\title{
RESEARCH
}

Open Access

\section{How COVID-19 lockdown and reopening affected daily steps: evidence based on 164,630 person-days of prospectively collected data from Shanghai, China}

Ding Ding ${ }^{1,2 \dagger}$, Minna Cheng ${ }^{3 \dagger}$, Borja del Pozo Cruz ${ }^{4}$, Tao Lin ${ }^{5}$, Shuangyuan Sun ${ }^{3}$, Li Zhang $^{5}$, Qinping Yang ${ }^{3}$, Zhicong Ma ${ }^{6}$, Jing Wang ${ }^{5}$, Yingnan $\mathrm{Jia}^{6,7^{*}}$ (i) and Yan Shi ${ }^{3,8^{*}}$

\begin{abstract}
Background: COVID-19 lockdowns may lead to physical inactivity, a major risk factor for non-communicable diseases. This study aims to determine: 1 ) the trajectory in daily step counts before, during and after the lockdown in China, and 2) the characteristics associated with the trajectories.

Methods: From December 2019 to July 2020, smartphone-based step counts were continuously collected in 815 Chinese adults residing in Shanghai over 202 days across three phases: before, during, and after the lockdown. Participant characteristics were reported, and height, weight and body composition measured before the lockdown. A 'sharp' regression discontinuity design with cluster robust standard errors was used to test the effect of the lockdown and reopening on daily steps and a linear mixed model was used to examine the characteristics associated with trajectories during the observed period.

Results: Based on 164,630 person-days of data, we found a sharp decline in daily step counts upon the lockdown $(24 / 01 / 2020)$ by an average of $3796(\mathrm{SE}=88)$ steps, followed by a significant trend of increase by 34 steps/day $(\mathrm{SE}=2.5 ; p<.001)$ until the end of the lockdown (22/03/2020). This increasing trend continued into the reopening phase at a slower rate of 5 steps per day $(S E=2.3 ; p=0.029)$. Those who were older, married, university educated, insufficiently active, had an 'at risk' body composition, and those in the control group, were slower at recovering step counts during the lockdown, and those who were older, married, without university education and with an 'at risk' body composition recovered step counts at a slower pace after the reopening.

Conclusions: Despite later increases in step counts, COVID-19 lockdown led to a sustained period of reduced physical activity, which may have adverse health implications. Governments and health professionals around the world should continue to encourage and facilitate physical activity during the pandemic.
\end{abstract}

\footnotetext{
* Correspondence: jyn@fudan.edu.cn; shiyan@scdc.sh.cn

${ }^{\dagger}$ Ding Ding and Minna Cheng contributed equally to this work.

${ }^{6}$ School of Public Health, Key Lab of Public Health Safety of the Ministry of Education, Fudan University, 130 Dongan Road, Shanghai 200032, China

${ }^{3}$ Shanghai Municipal Center for Disease Control and Prevention, 1380 West Zhongshan Road, Shanghai 200336, China

Full list of author information is available at the end of the article
} 


\section{Introduction}

First reported in Wuhan, China, in December 2019, Coronavirus disease-2019 (COVID-19) has quickly spread around the world, causing colossal health, social and economic damage. On March 11, 2020, the World Health Organization (WHO) declared COVID-19 a pandemic, [1] and by 30 Sep 2020, more than 33 million confirmed cases have been reported in more than 200 countries, resulting in over a million deaths worldwide [2]. To contain the spread of the virus, most governments around the world have adopted lockdowns and physical distancing measures. Such restrictions, although necessary for infection control, impose potential consequences in other areas of public health, such as cancer screening, [3] domestic violence, [4] mental health problems [5] and chronic disease management [6].

Of all the risk factors potentially affected by COVID19 lockdowns, physical inactivity is among the most studied. Mounting evidence supports the role physical activity plays in the prevention and management of chronic illness, such as coronary heart disease, type 2 diabetes and some cancers [7, 8]. A recent study based on the large UK Biobank cohort also found that regular physical activity may protect against COVID-19 hospitalisation, an indicator of severe symptoms [9]. Since initial lockdown restrictions have been implemented, several online surveys have been conducted to examine how physical activity levels changed as a result of the lockdown. A multi-country survey found a significant reduction in all intensity levels of physical activity during home confinement [10]. Similar reductions in physical activity have been confirmed by studies in Spain, [11] Australia [12] and Chile, [13] while a study in Belgium found an increase in exercise frequency [14]. While these studies provide useful insights into the effects of COVID-19 lockdowns on physical activity, they are subject to several limitations: first, nearly all studies are based on cross-sectional or retrospective data collections, without having a 'real' baseline prior to the lockdown; second, these studies are based on self-reported physical activity only, which is subject to measurement bias; third, except for a few studies that examined subgroup differences, $[15,16]$ most studies only reported the overall change in physical activity without investigating the characteristics associated with change in physical activity; fourth, nearly all studies examined the effects of the lockdown only without investigating the effects of reopening. The only way to avoid these major limitations is analysis of retrospectively obtained device data, which was done by one study to our knowledge [17]. This study found world-wide declines in step counts following the COVID-19 lockdown. While this descriptive study has established the feasibility of examining the effects of the lockdown on smartphone-derived step counts, it was based on aggregated data without providing any information regarding the smartphone users, nor did it examine individual characteristics associated with step count trajectories during the lockdown.

In the current study, we aimed to address several major limitations described above by using device-based, prospectively collected, continuous monitoring of daily step counts of a cohort of Chinese adults living in Shanghai spanning over 200 days across different phases of the COVID-19 pandemic. Our objectives are to determine: 1) the change in daily steps in response to the lockdown and reopening during the COVID-19 pandemic in China, and 2) the characteristics associated with trajectories in step counts during and after the lockdown. Findings from the study will not only help evaluate effects of COVID-19 restrictions on populationlevel physical activity, but also identify the subpopulations at risk of a sedentary lifestyle during the pandemic to inform preventive intervention strategies.

\section{Methods \\ Sample and procedures}

The study was conducted in Shanghai, China, an economically advanced city in East China with a population of more than 27 million people. We used step count data of a sample of 815 Chinese adults who participated in a 13-week step intervention which took place between September and November 2019. Participants were sampled from 11 workplaces in Pudong District, Shanghai. Participants were eligible if they were aged 18 years and above, without major chronic disease or disability, not pregnant, and without any intention to leave the current workplace in 12 months. Following the completion of the intervention on $30 / 11 / 2019$, participants entered a nine-month follow-up phase which allows the research staff to access their step count data until 31/08/ 2020. Although the intervention group had higher step counts upon the completion of the intervention, by 20/ $12 / 19$, the difference between groups had substantially reduced. Therefore, we consequentially used both the intervention and control groups as a cohort for continuous step tracking (Supplementary Fig. 1). All study procedures have been approved by the Shanghai Municipal Center for Disease Control and Prevention Ethical Review Committee (ChiCTR1900023813) and all participants in the study provided written informed consent before taking part in the study.

\section{Measures \\ Step counts}

Daily step count was measured by WeRun, a social fitness plugin built in WeChat, the most popular mobile social media application in China. WeRun imports step count data from a smartphone's built-in accelerometer, [18] and 
during the study participants shared their daily step counts via a cloud-based secure server. Based on a pilot study conducted prior to the intervention, step counts according to WeRun correlated strongly (spearman correlation coefficient $0.766, p<0.001$ ) with step counts measured by a hip-worn accelerometer (Actigraph GT3xBT). We considered days with less than 1000 steps as an invalid wearing day (Supplementary Table 1) and daily step counts were truncated at 30,000 steps/day.

Baseline characteristics of participants were collected by trained research assistants. Participants reported demographic characteristics, including birth year and month, sex, marital status, educational attainment, and income, through a self-administered questionnaire. A trained interviewer was present during the completion of the questionnaire in case of questions and to ensure the completion of the questionnaire. Baseline physical activity was measured using the validated Chinese version of the International Physical Activity Questionnaire short form [19] and was categorised as sufficiently active ( $\geq 1000$ metabolic equivalent [MET] minutes/ week) vs insufficiently active $(<1000$ MET minutes/ week) [20]. We used the cut point that approximates the upper end of the current recommendations, [7] because the participants were overall highly active. Adiposity was measured using body mass index (BMI, calculated from measured height and weight (TCS-150 electric scale and Omron HBF-214 Body Composition Monitor Scale), categorised using Asian-specific cut-points as lower risk $(<23)$, increased risk (23-27.4), and high risk $\geq$ (27.5) [21]) and body fat percentage using the reliable and valid [22] Omron HBF-214 Body Composition Monitor Scale ( $\leq 20$ vs $>20$ for men and $\leq 30$ vs $>30$ for women [23]).

\section{Timeline}

Located more than $800 \mathrm{~km}$ East of Wuhan, Shanghai has been moderately affected by the pandemic. As of 31/08/ 2020, Shanghai recorded a total of 903 cases and 7 deaths. On 24/01/2020, following the Central Government's Public Health Emergency Response Policies, Shanghai enforced 'Level 1 (the highest level of) Restrictions' with residents ordered to stay at home and all public places closed. The start of the COVID-19 Pandemic overlapped with the Chinese New Year, when most people were expected to gather with family and friends. Therefore, the initial two weeks of the lockdown was the most stringent. Between 10/02/2020 (the end of the Chinese New Year holiday) and 22/03/2020, some essential workers had returned to work while others continued to work from home for longer periods, and essential travels (e.g, grocery shopping) were allowed but controlled. Meanwhile, some public facilities and spaces gradually started to reopen. On 23/03/2020, Shanghai Government officially downgraded restrictions from
Level 1 to Level 2. By then, most employees had returned to work and public spaces had started to reopen with control measures, such as extensive cleaning, temperature check, and crowding prevention. Acknowledging the gradual process of the reopening, we chose $22 / 03 / 2020$ as 'the end of the lockdown' as this date represents an official down-grading of restrictions and returning to 'normal life'. Throughout the paper, we refer to the period before $24 / 01 / 2020$ as 'before the lockdown', the period between 24/01/2020 and 22/03/2020 as 'during the lockdown' and the period between 23/03/ 2020 and 08/07/2020 (last day of step data) as 'after the lockdown' or 'reopening'.

\section{Statistical analysis}

We linked step count data with baseline survey and biometric measures. For Research Aim 1 (change in step counts following the lockdown), we plotted the average daily step counts and the $95 \%$ confidence interval across the entire study period, for the entire sample and separately by the intervention and control groups. We used a 'sharp' regression discontinuity design (RDD) with cluster robust standard errors and binsize $=1$ day to test the effect of the lockdown on participants' step counts [24]. RDD is a causal inference method to evaluate whether an 'abrupt' change is causal by an intervention (i.e., the lockdown), by comparing the outcomes (i.e., step counts) following the intervention with the counterfactual without the intervention. Through minimising unobserved confounding, this quasi-experimental approach is designed to be analogous to a randomised control trial [24]. We used the Imbens and Kalyanaraman (IK-BW) method to determine the optimal bandwidth (i.e. number of days around either side of the lockdown date) for estimates of 'local' effect [25]. Based on the IK-BW method, we selected the bandwidth to be 26 days around the lockdown date (i.e., 13 days before and 13 days after). As a standard sensitivity analyses, we repeated the analysis using half ( 12 days, 6 days before and 6 days after) and double the bandwidth (50 days, 25 days before and 25 days after) around the lockdown date [25]. We conducted the RDD analysis for the start of the lockdown only, as the easing of lockdown was a more gradual process and there were no 'abrupt' changes in step counts.

For Research Aim 2 (determine characteristics associated with trajectories in step counts), we first built a linear mixed model with participants as a cluster variable, period (i.e., pre-lockdown, lockdown, and post-lockdown) and day as independent variables and step count as the dependent variable. We then tested a two-way interaction term (day $\mathrm{x}$ period) in the model to test whether step counts trajectories differed by period (i.e., comparing the lockdown and reopening periods with pre-lockdown as 
the reference). Finally, we tested whether baseline physical activity levels, adiposity (BMI and body fact percentage) and selected demographic characteristics (age: under $40 \mathrm{vs}$ 40+ years, sex, marital status: married vs unmarried/divorced/widowed, education: university vs no university education) moderated the change in step count trajectory by testing a three-way interaction term (day $\mathrm{x}$ period $\mathrm{x}$ covariate of interest-e.g., baseline adiposity). All models were adjusted for relevant covariates (i.e., age, sex, education, income, marital status, worksite, intervention group allocation, baseline physical activity and baseline adiposity). We used $\mathrm{R}$ software for all our computations. The significance level was set at $p$-value 0.05 , two-tailed. We provide an example of the codes used to run all the analysis in supplementary files.

\section{Results}

Participant characteristics are summarised in Table 1. Averaged 40 years of age, nearly two thirds of the

Table 1 Participants characteristics at baseline $(n=815)^{a}$

\begin{tabular}{|c|c|}
\hline Variables & n (\%)* \\
\hline \multicolumn{2}{|l|}{ Age (year) } \\
\hline $20-29$ & $117(14.4)$ \\
\hline $30-39$ & $309(38.1)$ \\
\hline $40-49$ & $259(31.9)$ \\
\hline $50+$ & $126(15.5)$ \\
\hline \multicolumn{2}{|l|}{ Sex } \\
\hline Male & $285(35.0)$ \\
\hline Female & $530(65.0)$ \\
\hline \multicolumn{2}{|l|}{ Education } \\
\hline High school graduate or below & $161(19.9)$ \\
\hline Vocational training & $154(19.0)$ \\
\hline University or higher & $496(61.2)$ \\
\hline \multicolumn{2}{|l|}{ Marital status } \\
\hline Married & $672(83.3)$ \\
\hline Single/divorced/widowed & $135(16.7)$ \\
\hline \multicolumn{2}{|l|}{ Body Mass Index (kg/m2) } \\
\hline Low risk $(<23)$ & $387(47.8)$ \\
\hline Increased risk (23-27.4) & $318(39.3)$ \\
\hline High risk $(\geq 27.5)$ & $105(13.0)$ \\
\hline \multicolumn{2}{|l|}{ Body fat percentage } \\
\hline Lower risk ( $\leq 20 \%$ for men and $\leq 30 \%$ for women) & $263(32.8)$ \\
\hline Higher risk (> 20\% for men and > 30\% for women) & $539(67.2)$ \\
\hline \multicolumn{2}{|l|}{ Baseline physical activity levels } \\
\hline Insufficient (<1000 MET ${ }^{\mathrm{b}}$ minutes/week) & $256(33.2)$ \\
\hline Sufficient ( $\geq 1000 \mathrm{MET}^{\mathrm{b}}$ minutes/week) & $514(66.8)$ \\
\hline
\end{tabular}

participants were female, more than $60 \%$ had a university degree or higher, and more than four in five were married. At baseline, slightly less than half of the participants were classified as normal weight, nearly $40 \%$ overweight and 13\% obese based on BMI risk classification specific to Asian populations [21]. Based on body composition, more than two thirds were classified 'at risk'. Participants had high levels of physical activity overall with two thirds reporting at least 1000 MET minutes of physical activity per week. Compared with a previous surveillance survey among a population-representative sample of working-age adults in Shanghai, [26] participants from the current study have higher education and reported higher levels of physical activity.

Figure 1 showed average daily steps over 202 days for the entire sample (data for the intervention and control groups separately were presented in Supplementary Fig. 2). Overall, prior to the lockdown, the daily step counts averaged above 8000, which remained stable without statistically significant changes until the day before the lockdown $(p=0.073)$. On the first day of the lockdown (24/01/2020, Day 36 of the study period), the average daily step counts declined abruptly by 3796 steps $(\mathrm{SE}=88)$. Results from RDD analysis (Fig. 2) suggested a sharp drop in step counts because of the lockdown $(p<.001)$. Sensitivity analyses with half and double chosen bandwidths confirmed these results (Table 2). Since the initial decline, there was a significant trend in step count increase by 34 steps per day $(\beta=34.1 ; \mathrm{SE}=$ 2.5; $p<.001)$. This increasing trend continued until the end of the lockdown (23/03/2020, Day 95) where the daily step counts recovered to only 708 steps $(\mathrm{SE}=$ 85) lower than on Day 1. Since then and until the end of our study period (09/07/2020, Day 202), daily step counts continued to increase by 5 steps per day $(ß=5.0 ; \mathrm{SE}=2.3 ; p=0.029)$.

We tested whether the trend in daily steps during and after the lockdown differed by baseline characteristics and found that age, education, marital status, intervention allocation, baseline physical activity and body composition significantly modified step count trajectories (Table 3). Specifically, the increase in steps during the lockdown was attenuated in those aged 40 years and above $(B=-20.5 ; \mathrm{SE}=5.0 ; p<0.001$; interpreted as 20.5 fewer steps of increase per day compared with those aged below 40 years, those who were married $(B=-22.6$; $\mathrm{SE}=6.9 ; p<0.001)$, those with university education $(B=$ -23.8 ; $\mathrm{SE}=5.2 ; p<0.001)$, those who were in the control group of the previous intervention $(B=-24.9 ; \mathrm{SE}=5.2$; $p<0.001)$, those who were insufficiently active at baseline $(B=-27.5 ; \mathrm{SE}=5.3 ; p<0.001)$, and those with a body composition classified as 'at risk' $(\beta=-27.8$; $\mathrm{SE}=$ 5.4; $p<0.001)$. During the reopening phase, the increase in step counts was again attenuated in those aged 40 


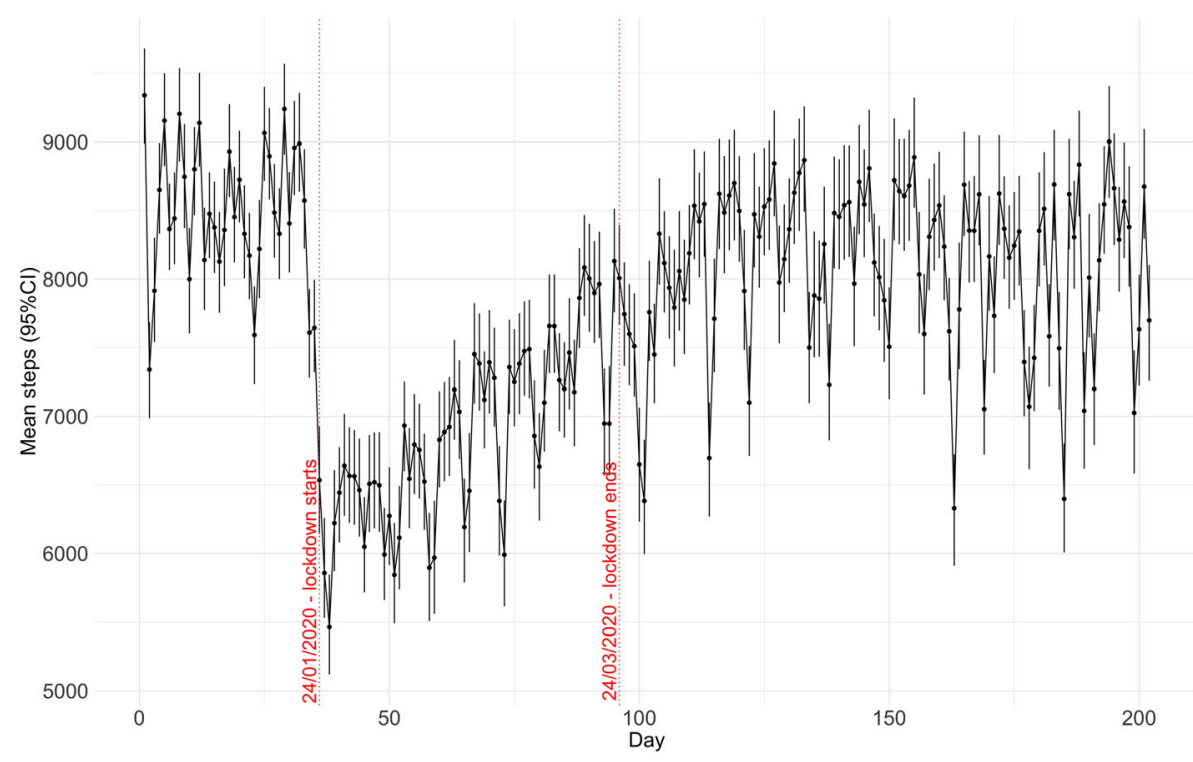

Fig. 1 Mean daily steps (95\% Cl) of participants before, during and after the lockdown

years and above $(\beta=-11.1 ; \mathrm{SE}=2.3 ; p=0.02)$, those who were married $(\beta=-15.9 ; \mathrm{SE}=6.3 ; p=0.01)$, and those with 'at risk' body composition $(ß=-18.0$; $\mathrm{SE}=$ $4.9 ; p<0.001)$. However, after the lockdown restrictions were eased, those with university education had significantly larger increases in steps instead $(ß=11.9$; $\mathrm{SE}=$ 4.8; $p=0.013)$, but there was no longer a difference in step count trajectories by baseline physical activity levels $(ß=-6.0 ; \mathrm{SE}=4.9 ; p=0.22)$. We did not find significant effect modification by sex or BMI categories during or after the lockdown. For data visualisation, we stratified step count trajectories by significant effect modifiers during and after the lockdown (Fig. 3).

\section{Discussion}

In this study, we used prospectively collected step count data over 202 days and 164,630 person-days to examine the effects of COVID-19 lockdown restrictions had on physical activity in Shanghai, China. To our knowledge, this is the first prospective study on the effects of COVID-19 lockdown and reopening on an objectively measured physical activity outcome. Our study revealed potential longer-term consequences of lock-down restrictions on daily step counts and identified specific population subgroups that may be particularly susceptible to declines in physcial activity, such as those who were older, married, insufficiently active at baseline and those with 'at risk' body composition.

Findings from our study extended evidence from previous survey-based studies of physical activity declines following the COVID-19 lockdown restrictions. For example, based on an online survey of 1491 adults in Australia, conducted in April 2020, 49\% of the participants reported declined physical activity, while $21 \%$ reported increased physical activity [12]. Similarly, an online survey of 2381 Polish adults, conducted in April and May 2020, reported that $43 \%$ of participants decreased physical activity while only $19 \%$ increased [27]. Additionally, a Sport England survey conducted in April 2020 found that $41 \%$ of adults reported doing less physical activity while $31 \%$ reported doing more [28]. A Spanish study of 72 type 2 diabetes patients conducted in April and May 2020 found that participants' weekly walking time declined from more than $300 \mathrm{~min}$ to just over $100 \mathrm{~min}$ [11]. These studies are subject to recall bias because data were collected retrospectively in response to the lockdown, by asking participants to compare activity levels before and after the lockdown. Such

Table 2 Effects of the lockdown on step counts of participants $(n=815)$

\begin{tabular}{llll}
\hline Bandwidth & Observations (person-day) & $\boldsymbol{\beta}$ (SE) (steps/day) & $\boldsymbol{p}$-value \\
\hline IK-bandwidth & 32,424 & $-2158(126.20)$ & $<.001$ \\
Half- bandwidth & 15,973 & $-1839(139.38)$ & $<.001$ \\
Double- bandwidth & 54,374 & $-2253(120.87)$ & $<.001$ \\
\hline
\end{tabular}

IK, Imbens and Kalyanaraman; SE, Standard Error

Models are adjusted for age, sex, education, income, marital status, intervention group allocation, baseline physical activity and body mass index

All models use robust standard errors with participant ID as a cluster unit 


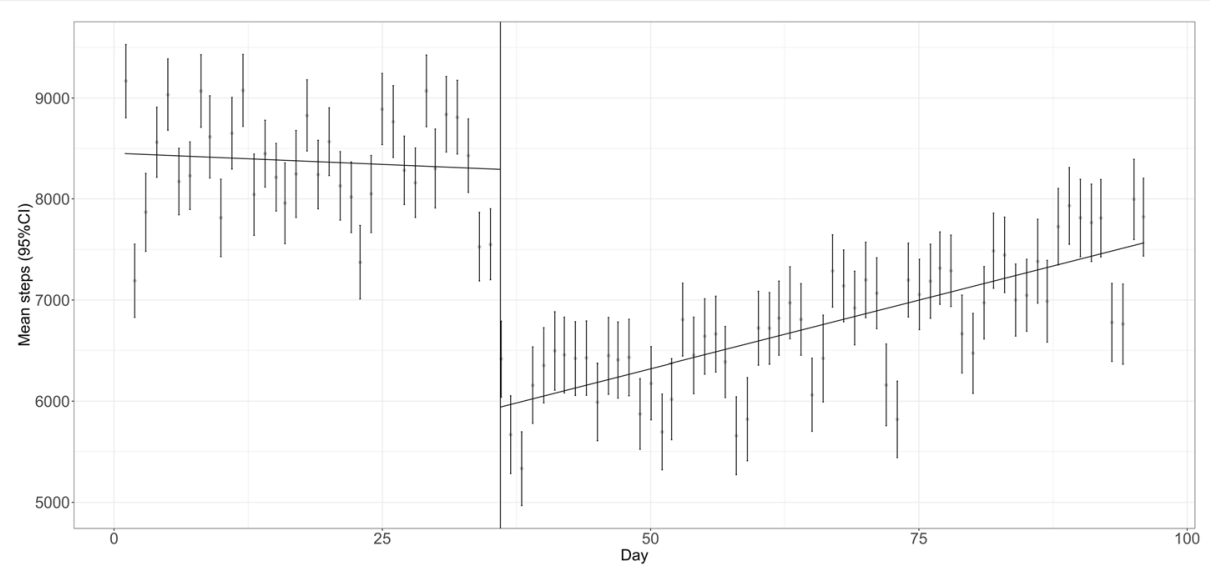

Fig. 2 Graphic display of regression discontinuity analysis

data could be informative and may be the only data that could be collected in most circumstances, considering the short notice usually given ahead of a lockdown and the amount of time required to obtain ethical approval and to design and conduct a study. In comparison, our study was opportunistic as COVID-19 happened during our ongoing data collection, therefore we could collect 'true' baseline data and follow up participants over time. Our findings, based on prospectively collected objective data, mostly confirmed findings from survey-based cross-sectional and retrospective studies.

Another advantage of our study is continuous data collection over 202 days, which provided a unique opportunity to examine the trajectory of step counts through different phases: before, during, and after the lockdown. Based on our data, there seemed to be a sharp initial drop in daily step counts by more than $40 \%$, followed by slow but steady increase. This sharp decline may be a result of the abrupt nature of the lockdown.
On 23/01/2020, the Chinese Government imposed a strict lockdown in Wuhan and banned all travels. By 24/01/ 2020, the local governments of 17 provinces (Shanghai included) implemented First-Level Public Health Emergency Response Policies. The date of the lockdown was particularly chosen to prevent social gatherings ahead of the Lunar New Year (starting 25/01/2020). In China, Lunar New Year is a public holiday when employees and students return home and it is a cultural tradition to visit families and friends and to gather for feasts and celebration. The Lunar New Year Holiday in 2020 was extended due to the pandemic. Therefore, the sharp decline in daily steps was a result of nearly everyone staying at home as a result of both the Lunar New Year and the lockdown. While step counts remained low for around two weeks, we observed slow but steady increase in step counts since then. This trajectory may be explained by the gradual process of returning to work. In Shanghai, the initial reopening of some workplaces and the resumption of the

Table 3 Test of effect modification: differences in the trajectory of daily step counts before, during, and after the lockdown ( $n=815$ )

\begin{tabular}{|c|c|c|c|c|}
\hline & \multicolumn{2}{|c|}{ During lockdown } & \multicolumn{2}{|c|}{ After lockdown } \\
\hline & $\beta(\mathrm{SE})^{\mathrm{a}}$ & $p$-value & $\beta$ (SE) $^{a}$ & $p$-value \\
\hline Age (40+ vs 20-39years) & $-20.46(5.02)$ & $<.001$ & $-11.07(4.61)$ & 0.016 \\
\hline Sex (female vs male) & $-2.75(5.29)$ & 0.602 & $0.31(4.86)$ & 0.947 \\
\hline Martial status (married vs not married) & $-22.62(6.90)$ & 0.001 & $-15.89(6.33)$ & 0.012 \\
\hline Education (university vs no university) & $-23.80(5.24)$ & $<.001$ & $11.88(4.81)$ & 0.013 \\
\hline Baseline physical activity (insufficient vs sufficient) & $-27.52(5.33)$ & $<.001$ & $-6.01(4.89)$ & 0.219 \\
\hline BMI (overweight/obese vs normal weight) & $-8.81(5.03)$ & 0.079 & $-9.05(4.62)$ & 0.050 \\
\hline Body composition (at risk vs not at risk) & $-27.82(5.35)$ & $<.001$ & $-17.95(4.91)$ & $<.001$ \\
\hline Intervention group allocation (control vs intervention) & $-24.94(5.17)$ & $<0.001$ & $-3.69(4.75)$ & 0.437 \\
\hline
\end{tabular}

${ }^{a}$ Interpreted as the differences in the change of daily step counts. For example: those aged $40+$ years increased step counts by 20.46 fewer steps than those aged $20-39$ years during the lockdown period

${ }^{\mathrm{b}}$ Test of effect modification was conducted in a linear mixed model with participants as a cluster variable, period (i.e., pre-lockdown, lockdown, and postlockdown) and day as independent variables and step count as the dependent variable. Effect modification was tested one at a time adjusted for age, sex, education, income, marital status, worksite, intervention group allocation, baseline physical activity and baseline adiposity 

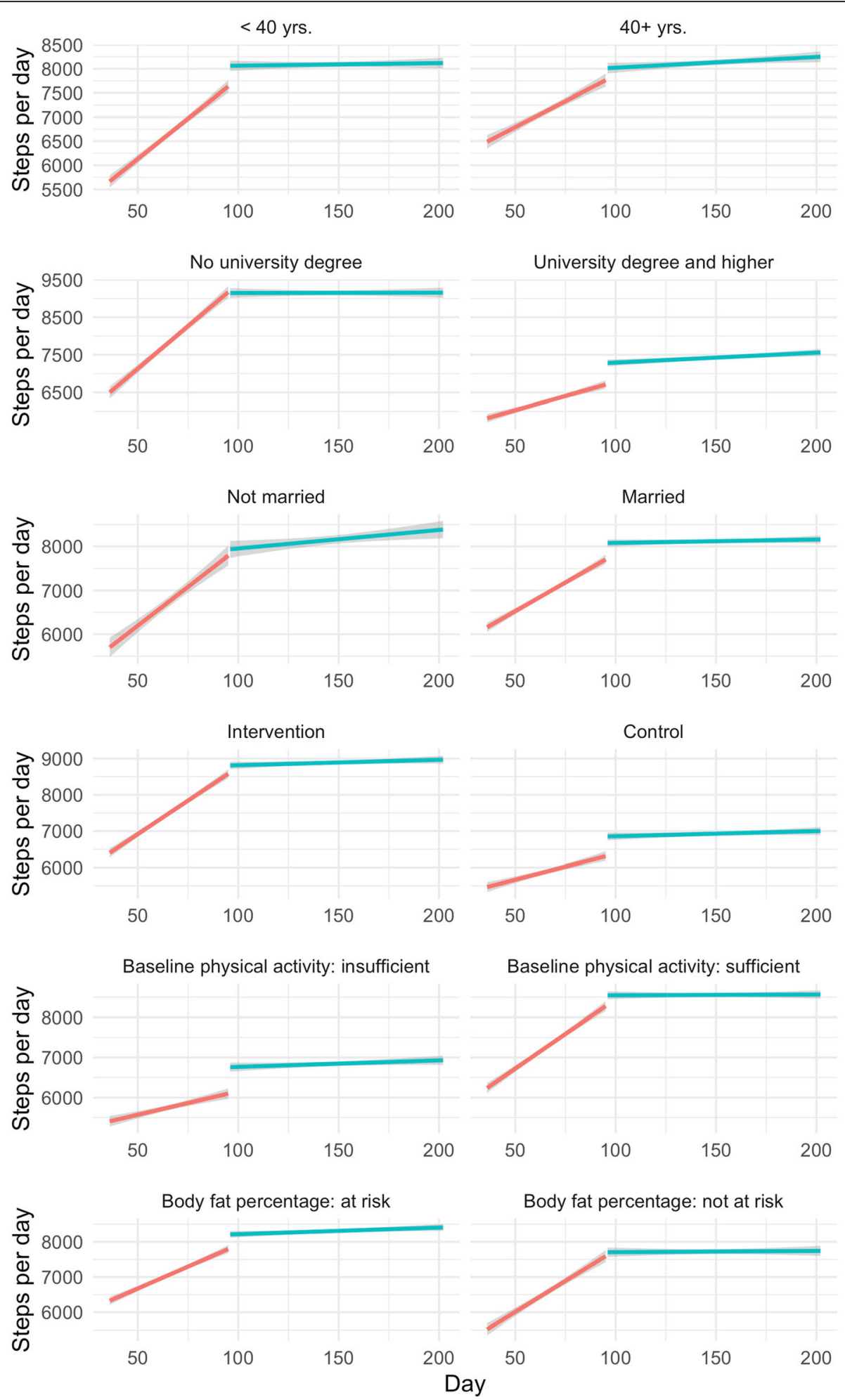

Fig. 3 Unadjusted stratified trajectory of steps counts during and after the lockdown

public transport system started on 10/02/2020. By 29/02/ 2020, nearly two thirds of all employees had returned to work. Although step counts continued to increase towards and beyond 23/03/2020 when the restrictions were officially eased, by the end of the study, 3.5 months into the reopening, the total step counts were still slightly below 
the baseline level. This finding may reflect people's caution and hesitation that things have not returned $100 \%$ to normal.

According to our findings, lockdown restrictions affected some individuals' step counts more than others. Previously, only a few studies examined the characteristics associated with retrospectively reported change in physical activity following the COVID-19 lockdown [12, 14-16]. Specifically, Reyes-Olavarria et al. found that those selfreported to be overweight were more prone to decreased physical activity [13]. We examined adiposity both in terms of measured BMI and body composition and found the latter to be a significant effect modifier both during and after the lockdown. Increasing evidence suggests that adiposity may be a predictor of physical activity declines $[29,30]$. Our finding echoed this observation within the 'unnatural' context of lockdown restrictions. In terms of baseline physical activity, findings from our study suggest that in China, those who were more active at baseline recovered their daily step count more quickly after the initial decline. Such patterns continued beyond the reopening, implying that lockdown restrictions may disproportionately affect those who were less active. Furthermore, those in the intervention group, who started at a higher step count level than those in the control group, recovered their steps faster during, but not after, the lockdown. This difference may be explained by a sustained effect of the intervention, or the overall higher levels of physical activity among participants in the intervention group. Lesser et al. found that insufficiently active individuals were more likely to reduce physical activity than their active counterparts, [16] but Constandt et al. found the opposite - that those who were less active before COVID-19 were more likely to increase activities during the lockdown [14]. The role habitual physical activity plays in activity change during the lockdown may be context-specific. For example, outdoor exercise was permitted as 'essential activity' by some governments, such as those in Australia and the UK, but banned in Shanghai, China, between 24/01/2020 and 23/03/2020. Our recent analysis of Google query data suggested an unprecedented surge in online searches for exercise, particularly home-based exercise during the COVID-19 lockdown [31]. This suggests that lockdown restrictions may have prompted people to form new exercise habits, whether initiating exercise or adapting exercise routines according to restrictions. Interestingly, Constandt et al. found that $61 \%$ of those who were habitually inactive at baseline reported that there was more time to exercise during the lockdown, and those who were used to exercising with friends or in a club were more likely to reduce physical activity during the lockdown, despite being highly active at baseline [14]. This suggests that specific types of physical activity individuals were used to doing prior to the lockdown, their social context and adaptability to an indoor environment may determine ones' ability to maintain physical activity during the lockdown.

Our study also identified some demographic characterises predictive of the trajectory in physical activity during and after the lockdown. For example, those who were older (40+ years) recovered step counts at a slower pace than their younger counterparts. This may be explained by the higher perceived susceptibility to COVID-19 and other competing priorities, such as home-schooling children, experienced in this age group. Furthermore, older Chinese adults typically exercise outdoors (e.g., Taichi, square dance) with social groups, [32] and may be more affected by lockdown restrictions than their younger counterparts. Our study also found that those who were married experienced a slower recovery in step counts than their unmarried counterparts. While this could also be a result of competing priorities, such as home-schooling or household chores, this pattern could also be an artefact of the lockdown policies. Between $24 / 01 / 2020$ and $22 / 03 / 2020$, the lockdown restrictions included reducing non-essential travel and limiting essential shopping trips to one per household per day in most communities. Therefore, unmarried adults and smaller households would receive more allowed opportunities for travel per person than married adults and larger households. Finally, the role education played was particularly interesting: while university-educated adults increased steps slower during the lockdown, they recovered steps faster after the lockdown. A possible explanation is that university-educated adults were more likely to be knowledge workers who had the privilege to work from home during the lockdown, and they may also have more financial resources to use private cars or taxis when they needed to travel. According to a recent Chinese national travel survey, during the COVID-19 pandemic, car and taxi trips accounted for $45 \%$ of all trips compared with $5.8 \%$ before the pandemic [33]. This change in modal share may have led to a reduction in incidental walking, particularly in those who can afford avoiding public transport.

\section{Strengths and limitations}

Compared with other existing studies, our study has several important strengths, such as prospective data collection, objectively measured step counts, and continuous data of 164,630 person-days, spanning across 7 months before, during and after the lockdown. We also used innovative causal inference methods to confirm that the association between lockdown and step decline was causal. However, several limitations need to be acknowledged. First, we opportunistically used long-term follow-up data of a step count intervention. Although our baseline phase started after the between-group 
difference in step counts returned to null, we cannot be certain whether our baseline step counts were inflated as a result of the intervention. Second, we used smartphone-based accelerometers for measuring step counts, which can be subject to participants not wearing the phone consistently throughout the day. Particularly, smartphones may systematically underestimate homebased activities because participants are less likely to carry their phones continuously at home (as shown by data presented in Supplementary Table 1). Finally, because we opportunistically used a sample of intervention participants, which was not intended to be populationrepresentative, findings from the current study have limited generalisability.

\section{Implications}

Based on objectively measured step counts over 164,630 person-days, our study revealed a sharp decline in daily step counts immediately following the COVID-19 lockdown in Shanghai, China. Despite later increases in step counts during and after the lockdown, such a sustained period of lower physical activity could still have health implications, [34] such as metabolic derangements and change in body composition [35]. Considering such potential health consequences and the critical role physical activity plays in improving immune function, [36] governments and health professionals around the world should continue to encourage and facilitate physical activity during the pandemic. Other sectors, such as fitness/recreation and transportation, should use COVID-19 as an opportunity to upgrade service and management standard [37] and transform the current practice [38].

\section{Abbreviations}

COVID-19: Coronavirus disease-2019; WHO: World Health Organization; MET: metabolic equivalent; BMI: body mass index; RDD: regression discontinuity design; IK-BW: Imbens and Kalyanaraman; SE: standard error

\section{Supplementary Information}

The online version contains supplementary material available at https://doi. org/10.1186/s12966-021-01106-X.

\section{Additional file 1}

\section{Acknowledgements}

Our special thanks to the participants for their time and the worksite organisers

\section{Authors' contributions}

YJ, MC and DD conceptualised the idea, YJ MC and YS obtained funding, BdPC conducted data analysis, DD drafted the paper and all authors participated in reviews and interpretation of analysis results and critical revision of the paper. All authors approved the final version for submission.

\section{Funding}

The study was funded by a grant awarded by the National Natural Science Foundation of China (NSFC; 72004027) and Shanghai Municipal Health Commission (GWV-7 \& GWV-10.1-XK05). DD was funded by a Heart Foundation Future Leader Fellowship (\#101234) and a Sydney Research Accelerator
(SOAR) Prize. The funders had no role in study design, data collection and analysis, decision to publish, or preparation of the manuscript.

Availability of data and materials

The datasets are available from the corresponding author on reasonable request.

\section{Declarations}

Ethics approval and consent to participate

All study procedures have been approved by the Shanghai Municipal Center for Disease Control and Prevention Ethical Review Committee (ChiCTR1900023813) and all participants in the study provided written informed consent before taking part in the study.

\section{Consent for publication}

Not applicable.

\section{Competing interests}

There was no conflict of interest to declare from any authors.

\section{Author details}

${ }^{1}$ Prevention Research Collaboration, Sydney School of Public Health, Faculty of Medicine and Health, The University of Sydney, Camperdown, New South Wales, Australia. ${ }^{2}$ Charles Perkins Centre, The University of Sydney, Camperdown, New South Wales, Australia. ${ }^{3}$ Shanghai Municipal Center for Disease Control and Prevention, 1380 West Zhongshan Road, Shanghai 200336, China. ${ }^{4}$ Centre for Active and Healthy Ageing, Department of Sports Science and Clinical Biomechanics, University of Southern Denmark, Campusvej 55, 5230 Odense, Denmark. ${ }^{5}$ Shanghai Pudong New Area Center for Disease Control and Prevention, Shanghai 200136, China. ${ }^{6}$ School of Public Health, Key Lab of Public Health Safety of the Ministry of Education, Fudan University, 130 Dongan Road, Shanghai 200032, China. ${ }^{7}$ Health Communication Institute, Fudan University, Shanghai 200032, China.

${ }^{8}$ National Clinical Research Center for Aging and Medicine, Huashan Hospital, Fudan University, Shanghai 200040, China.

Received: 20 October 2020 Accepted: 4 March 2021

Published online: 17 March 2021

\section{References}

1. World Health Organization. WHO Director-General's opening remarks at the media briefing on COVID-19 - 1120202020 [Available from: https://www. who.int/dg/speeches/detail/who-director-general-s-opening-remarks-at-themedia-briefing-on-covid-19\%2D\%2D-11-march-2020.

2. World Health Organization. WHO Coronavirus Disease (COVID-19) Dashboard 2020 [cited 2020 01/10]. Available from: https://covid19.who.int/.

3. Maringe C, Spicer J, Morris M, Purushotham A, Nolte E, Sullivan R, et al. The impact of the COVID-19 pandemic on cancer deaths due to delays in diagnosis in England, UK: a national, population-based, modelling study. The Lancet Oncol. 2020;21(8):1023-34.

4. Boserup B, McKenney M, Elkbuli A. Alarming trends in US domestic violence during the COVID-19 pandemic. Am J Emerg Med. 2020;38(12):2753-5.

5. Pfefferbaum B, North CS. Mental health and the Covid-19 pandemic. N Engl J Med. 2020;383(6):510-2.

6. Hartmann-Boyce J, Morris E, Goyder C, Kinton J, Perring J, Nunan D, et al. Diabetes and COVID-19: risks, management, and learnings from other National Disasters. Diabetes Care. 2020;43(8):1695-703.

7. Physical Activity Guidelines Advisory Committee. 2018 physical activity guidelines advisory committee scientific report. Washington, DC: U.S. Department of Health and Human Services; 2018.

8. Lee IM, Shiroma EJ, Lobelo F, Puska P, Blair SN, Katzmarzyk PT. Effect of physical inactivity on major non-communicable diseases worldwide: an analysis of burden of disease and life expectancy. Lancet. 2012;380(9838): 219-29.

9. Hamer M, Kivimaki M, Gale CR, Batty GD. Lifestyle risk factors, inflammatory mechanisms, and COVID-19 hospitalization: a community-based cohort study of 387,109 adults in UK. Brain Behav Immun. 2020;87:184-7.

10. Ammar A, Brach M, Trabelsi K, Chtourou H, Boukhris O, Masmoudi L, et al. Effects of COVID-19 home confinement on eating behaviour and physical 
activity: results of the ECLB-COVID19 international online survey. Nutrients. 2020;12(6):28.

11. Ruiz-Roso MB, Knott-Torcal C, Matilla-Escalante DC, Garcimartin A, Sampedro-Nunez MA, Davalos A, et al. COVID-19 lockdown and changes of the dietary pattern and physical activity habits in a cohort of patients with type 2 diabetes mellitus. Nutrients. 2020;12(8):04.

12. Stanton R, To QG, Khalesi S, Williams SL, Alley SJ, Thwaite TL, et al. Depression, anxiety and stress during COVID-19: associations with changes in physical activity, sleep, tobacco and alcohol use in Australian adults. Int J Environ Res Public Health. 2020;17(11):07.

13. Reyes-Olavarria D, Latorre-Roman PA, Guzman-Guzman IP, Jerez-Mayorga D, Caamano-Navarrete F, Delgado-Floody P. Positive and negative changes in food habits, physical activity patterns, and weight status during COVID-19 confinement: associated factors in the Chilean population. Int J Environ Res Public Health. 2020:17(15):28.

14. Constandt B, Thibaut E, De Bosscher V, Scheerder J, Ricour M, Willem A. Exercising in times of lockdown: an analysis of the impact of COVID-19 on levels and patterns of exercise among adults in Belgium. Int J Environ Res Public Health. 2020;17(11):10.

15. Gallo LA, Gallo TF, Young SL, Moritz KM, Akison LK. The impact of isolation measures due to COVID-19 on energy intake and physical activity levels in Australian University students. Nutrients. 2020;12(6):23.

16. Lesser IA, Nienhuis CP. The impact of COVID-19 on physical activity behavior and well-being of Canadians. Int J Environ Res Public Health. 2020;17(11):31.

17. Tison GH, Avram R, Kuhar P, Abreau S, Marcus GM, Pletcher MJ, et al. Worldwide effect of COVID-19 on physical activity: a descriptive study. Ann Intern Med. https://doi.org/10.7326/M20-2665.

18. Gui X, Chen Y, Caldeira C, Xiao D, Chen Y. When Fitness Meets Social Networks: Investigating Fitness Tracking and Social Practices on WeRun. Proceedings of the $2017 \mathrm{CH}$ Conference on Human Factors in Computing Systems; Denver, Colorado, USA: Association for Computing Machinery; 2017. p. 1647-59.

19. Macfarlane DJ, Lee CCY, Ho EYK, Chan KL, Chan DTS. Reliability and validity of the Chinese version of IPAQ (short, last 7 days). J Sci Med Sport. 2007; 10(1):45-51.

20. Jeong S-W, Kim S-H, Kang S-H, Kim H-J, Yoon C-H, Youn T-J, et al. Mortality reduction with physical activity in patients with and without cardiovascular disease. Eur Heart J. 2019:40(43):3547-55.

21. WHO Expert Consultation. Appropriate body-mass index for Asian populations and its implications for policy and intervention strategies. Lancet. 2004;363(9403):157-63.

22. Vasold KL, Parks AC, Phelan DML, Pontifex MB, Pivarnik JM. Reliability and validity of commercially available low-cost bioelectrical impedance analysis. Int J Sport Nutr Exerc Metab. 2019;29(4):406-10.

23. Wang C, Hou XH, Zhang ML, Bao YQ, Zou YH, Zhong WH, et al. Comparison of body mass index with body fat percentage in the evaluation of obesity in Chinese. Biomed Environ Sci. 2010;23(3):173-9.

24. Oldenburg CE, Moscoe E, Bärnighausen T. Regression discontinuity for causal effect estimation in epidemiology. Curr Epidemiol Rep. 2016;3(3):233-41.

25. Imbens $\mathrm{G}$, Kalyanaraman K. Optimal bandwidth choice for the regression discontinuity estimator. Rev Econ Stud. 2011;79(3):933-59.

26. Yan $\mathrm{Q}$, Yao H, Xu J, Cheng M, Shi Y, Zhong W. Influencing factors of physical inactivity among non-agricultural population in Shanghai in 2013. J Environ Occup Med. 2017;34(8):681-6.

27. Gornicka M, Drywien ME, Zielinska MA, Hamulka J. Dietary and lifestyle changes during COVID-19 and the subsequent lockdowns among polish adults: a cross-sectional online survey PLifeCOVID-19 study. Nutrients. 2020; 12(8):03.

28. Sport England. New exercise habits forming during coronavirus crisis. London, UK: Sport England; 2020.

29. Ekelund U, Kolle E, Steene-Johannessen J, Dalene KE, Nilsen AKO, Anderssen SA, et al. Objectively measured sedentary time and physical activity and associations with body weight gain: does body weight determine a decline in moderate and vigorous intensity physical activity? Int J Obes. 2017;41(12): 1769-74.

30. Bauman AE, Reis RS, Sallis JF, Wells JC, Loos RJ, Martin BW, et al. Correlates of physical activity: why are some people physically active and others not? Lancet. 2012;380(9838):258-71.

31. Ding D, Del Pozo CB, Green MA, Bauman AE. Is the COVID-19 lockdown nudging people to be more active: a big data analysis. Br J Sports Med. https://doi.org/10.1136/bjsports-2020-102575.
32. Liu Z, Kemperman A, Timmermans H. Social-ecological correlates of older adults' outdoor activity patterns. J Transp Health. 2020;16:100840.

33. Jiang N, Li S, Cao S, Wei J, Wang BB, Qin N, Duan XL. Transportation Activity Patterns of Chinese Population during the COVID-19 Epidemic [in Chinese]. Res Environ Sci. 2020;33:1675-82.

34. Mattioli AV, Ballerini Puviani M, Nasi M, Farinetti A. COVID-19 pandemic: the effects of quarantine on cardiovascular risk. Eur J Clin Nutr. 2020;74(6):852-5.

35. Bowden Davies KA, Sprung VS, Norman JA, Thompson A, Mitchell KL, Halford JCG, et al. Short-term decreased physical activity with increased sedentary behaviour causes metabolic derangements and altered body composition: effects in individuals with and without a first-degree relative with type 2 diabetes. Diabetologia. 2018;61(6):1282-94.

36. Nieman DC, Wentz LM. The compelling link between physical activity and the body's defense system. J Sport Health Sci. 2019;8(3):201-17.

37. Amagasa S, Machida M, Ding D, Inoue S. Preventing infectious diseases outbreaks at exercise facilities. Managing Sport Leisure. 2020:1-4.

38. Brooks JHM, Tingay R, Varney J. Social distancing and COVID-19: an unprecedented active transport public health opportunity. Br J Sports Med. https://doi.org/10.1136/bjsports-2020-102856.

\section{Publisher's Note}

Springer Nature remains neutral with regard to jurisdictional claims in published maps and institutional affiliations.
Ready to submit your research? Choose BMC and benefit from:

- fast, convenient online submission

- thorough peer review by experienced researchers in your field

- rapid publication on acceptance

- support for research data, including large and complex data types

- gold Open Access which fosters wider collaboration and increased citations

- maximum visibility for your research: over $100 \mathrm{M}$ website views per year

At $\mathrm{BMC}$, research is always in progress.

Learn more biomedcentral.com/submissions 